\title{
Research Paper: How People Emotionally Respond to the News on COVID-19: An Online Survey
}

\author{
Zahra Hamidein $^{1}$ D, Javad Hatami² ${ }^{\text {ID }}$, Tara Rezapour ${ }^{1 *}$ (D) \\ 1. Department of Cognitive Psychology, Institute for Cognitive Science Studies, Tehran, Iran. \\ 2. Department of Psychology, Faculty of Psychology and Education, University of Tehran, Tehran, Iran.
}

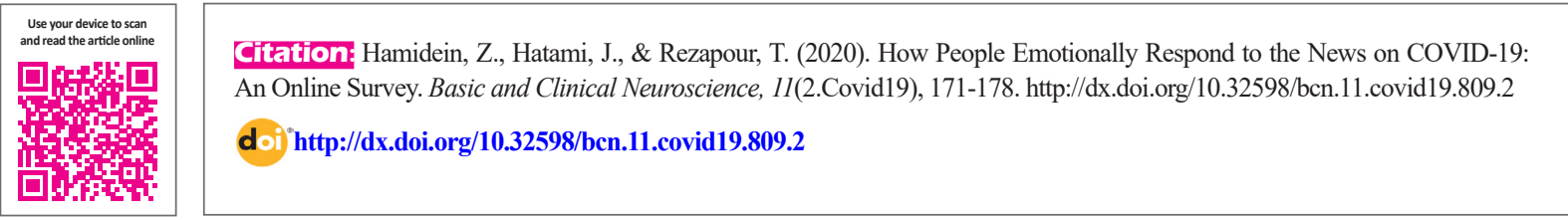

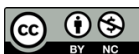

Article info:

Received: 10 Apr 2020

First Revision: 11 Apr 2020

Accepted: 13 Apr 2020

Available Online: 20 Apr 2020

Keywords:

COVID-19, Emotion, Emotion regulation, News

\begin{abstract}
A B S T RA C T
Introduction: During the Coronavirus Disease 2019 (COVID-19) outbreak, news media has played an important role in informing people to satisfy their curiosity about this stressful condition. Regular exposure to such stressful news may elicit different emotions in people and engage them in using strategies to control their emotions. In the present study, we aimed at exploring the most common negative emotion(s) experienced by individuals, as well as the most frequent Emotion Regulation (ER) strategies used facing the COVID-19-related news. We also examined whether the variable of personal relevance can moderate these emotional responses.

Methods: 617 individuals living in Tehran who regularly read the news about the COVID-19 from the early stages of spread completed an online survey. After excluding the participants with high scores from the Beck Depression Inventory ( $>18)$, data obtained from 443 participants were analyzed in terms of the experienced negative emotions and ER strategies.

Results: Anxiety (55.8\%) was the most common negative emotion reported by participants facing COVID-19-related news and problem-solving was the most frequent strategy used to control negative emotions. Both groups with high and low personal relevance indicated a similar pattern in experiencing high and low arousal emotions, as well as using ER strategies, and no significant differences were found $\left(\mathrm{X}^{2}=0.006, \mathrm{p}=0.51 ; \mathrm{X}^{2}=0.14, \mathrm{p}=0.39\right.$, respectively). We also found that participants with high scores in the resilience scale used an integrative rather than a single approach of the ER strategies $(\mathrm{rbp}=0.15, \mathrm{p}=0.01)$.

Conclusion: We found that during the COVID-19 outbreak, news media may have important role in triggering anxiety in people who regularly read the relevant news, and problem-solving was the most frequent strategy among them. Being directly involved with COVID-19 in personal life did not make any differences in the way that individuals emotionally respond to the news. While using an integrative approach in regulating emotion was found in more resilient individuals..
\end{abstract}

\footnotetext{
* Corresponding Author:

Tara Rezapour, PhD.

Address: Department of Psychology, Faculty of Psychology and Education, University of Tehran, Tehran, Iran.

Tel: +98 (912) 6185031

E-mail: rezapourt@iricss.org
} 


\section{Highlights}

- Anxiety was the common negative emotion experienced by the participants who regularly checked the COVID19-related news.

- Problem solving was the most frequent strategy used by the participants to regulate negative emotions.

- Being directly involved with COVID-19 in personal life did not make any differences in the way that individuals emotionally respond to the news.

- Using Integrative approach in regulating emotions was found in more resilient individuals.

\section{Plain Language Summary}

During the Coronavirus Disease 2019 (COVID-19) outbreak, news media has played an important role in providing people with latest updates on this stressful condition. Repeated exposure to such stressful news may elicit different emotions in people and engage them in using strategies to control their emotions. In this study, we aimed at exploring the most common negative emotion(s) experienced by individuals, as well as the most frequent Emotion Regulation strategies used facing the COVID-19-related news. We also examined whether the variable of personal relevance can moderate these emotional responses.

\section{Introduction}

uring the coronavirus disease 2019 (COVID-19) outbreak, mass media has played an important role in informing people, particularly during the early weeks of the crisis. Providing extensive negative news, including a high rate of morbidity and mortality caused by COVID-19, as well as uncertainties for preventive and therapeutic interventions, may evoke various emotional responses (Basch, Hillyer, Meleo-Erwin, Mohlma, Cosgrove, \& Quinones, 2020; Haroon \& Rizvi, 2020; Restubog, Ocampo, \& Wang, 2020). This issue has been addressed by previous studies establishing the association between viewing televised trauma (e.g. natural disasters, terrorisms, and disease outbreaks) and increased negative emotions, such as anxiety and sadness (Bodas, Siman-Tov, Peleg, \& Solomon, 2015; Szabo \& Hopkinson, 2007). Moreover, it has been reported that these emotional responses get even worse with constant contact with these news media (Bernstein, Ahern, Tracy, Boscarino, Vlahov, \& Galea, 2007).

Findings from previous studies indicate that emotional stimuli can be perceived as inherently relevant due to their possible impact on the organism's survival, particularly in threatening situations (Bayer, Ruthmann, \& Schacht, 2017; de Hoog \& Verboon, 2020; Smith \& Kirby, 2000). It means that, whenever individuals assess a stimulus or an event as relevant, their attention is automatically captured by the respective stimulus or event and leading to elicit augmented emotional responses (Bayer, Ruthmann, \& Schacht, 2017; Cooper \& Roter, 2000; de Hoog \& Verboon, 2020). Therefore, facing COVID-19 news, individuals may respond differently based on how much the news can affect their lives in the real world.

Given the importance of regulating emotions in face of emotional stimuli, several studies have considered different types of Emotion Regulation (ER) strategies and the adaptive use of these strategies in various situations (Boehme, Biehl, \& Mühlberger, 2019; Gutentag, Halperin, Porat, Bigman, \& Tamir, 2017; Lennarz, Hollenstein, Lichtwarck-Aschoff, Kuntsche, \& Granic, 2019). Emotion regulation is referred to as the extrinsic and intrinsic processes involved in monitoring, evaluating, and modifying emotional reactions, particularly in terms of intensity and temporal properties to achieve people's goals (Thompson, 1994). Emotions can be regulated using various strategies and different categories, such as primary control, secondary control, disengagement, and involuntary engagement (Lennarz et al., 2019). The use and selection of these strategies that may be influenced by personal- and contextual-related factors are of great importance (Kobylińska \& Kusev, 2019). Moreover, there is growing evidence emphasizing on flexibly using multiple regulation strategies to enable individuals to efficiently cope with changing contextual demands (Webb, Miles, \& Sheeran, 2012). 
Studies on ER are categorized into two groups, including experimental and field studies (English, Lee, John, $\&$ Gross, 2017). In experimental studies, participants are presented by emotional stimuli and instructed to use specific strategies to control their emotions to explore different outcomes (Butler, Egloff, Wlhelm, Smith, Erickson, \& Gross, 2003; Gross, 1998; Richards \& Gross, 2000). In the field studies, participants are observed to find how they select ER strategies to cope with different types of emotional experiences in everyday life (Doré, Silvers, \& Ochsner, 2016). Due to many uncontrollable variables outside of labs, the number of field studies is limited in this context. For example, in a study by Kashdan et al. (Kashdan, Goodman, Mallard, \& DeWall, 2016) using daily diary methodology, anger was the most intense emotion reported by participants that were regulated variously depending on its triggering factor. Another study (Brans, Koval, Verduyn, Lim, \& Kuppens, 2013) used an experience sampling method to identify the most frequently used ER strategies and their impacts on changing positive and negative affect in daily life. More recently, it has been reported that social aspects of the context may affect ER strategy selection in daily highpoints and low-points (English et al., 2017).

In addition to the important role of ER strategies that may enable individuals to efficiently cope with negative emotions, resilience is another factor that seems to be crucial in face of emotional conditions (Kay, 2016). The American Psychological Association (2014) defines resilience as "the process of adapting well in the face of adversity, trauma, tragedy, threats or even significant sources of stress (Southwick, Bonanno, Masten, Panter-Brick, \& Yehuda, 2014) (para. 4).” Previ) ous studies have shown that resilient people have more positive emotions and are more able to cope with negative emotional experiences (Block \& Kremen, 1996; Klohnen, 1996; Wolin \& Wolin, 1993). Therefore, the level of resilience could be reflected by an individual's ability to regulate their emotions, particularly the negative ones (Kay, 2016). However, there is no evidence that whether resilient individuals may differ in the use of ER strategies from non-resilient individuals.

Since the COVID-19 outbreak has resulted in excessive emotional experiences for individuals worldwide ( $\mathrm{Li}$, Wang, Xue, Zhao, \& Zhu, 2020), studies on the emotional-related issues in the context of real-life seem to be beneficial by providing more the real-world evidence. Moreover, due to different uncertainties regarding COVID-19, news media plays an important role to inform people with daily updates about different aspects of this crisis (Gao et al., 2020). Thus, during this extraordinary time, it can be interesting to be more informed about the emotional responses of people who regularly read the news and to find out which ER strategies they frequently use to manage their emotions. We also sought to determine whether high personal relevance to COVID-19-related news would make any differences in these regards.

\section{Method}

\subsection{Design and participants}

In this study, data were collected through an online survey using Google Forms. The sample size was considered a minimum of 375 subjects considering a population of over 5000 people, a confidence interval of $5 \%$, and a confidence level of $95 \%$ ). A specific form was then created, including a sharable link and was distributed through social media (Instagram and Telegram) over two weeks (2-16 May 2020). Participants were asked to answer the questionnaires after they signed a consent form. It should be noted that data were collected anonymously without asking the participant's name, and just in case of personal interest to be informed about the results, an optional section was considered for entering email addresses. The inclusion criteria were at least 18 years old, living in Tehran, and regularly (daily) reading the news on COVID-19 (from the early stages of spread). Participants with moderate to severe depression were excluded (score of $>18$ from the 21-item Beck Depression Inventory (BDI-21)).

\subsection{Measures}

The average time taken to complete the survey was 5-6 $\mathrm{min}$ and the questions were categorized into the following four sections:

Demographic data, including age, gender, educational level, the number of family members (including the respondent), and the mean of quarantine period (day) were assessed. In this section, participants were also asked about whether they were directly involved with issues associated with COVID-19 (as a person whose family was affected or died of COVID-19 or as a current medical staff member who was engaged in the fight against COVID-19) at the time of the survey. The latter question divided the participants into two groups, including with high personal relevance and low personal relevance.

Resilience was measured using the 14-item Resilience Scale (RS-14) developed by Wagnild and Young (Wagnild, 2009; Wagnild, \& Young, 1993). We used the Persian version of the scale that was validated by Hashemi 
et al. This version consists of 14 self-report items rated on a 5-point Likert scale ranging from ' 1 (strongly disagree) to 5 (strongly agree). The total score ranges from 14 to 70 , with higher scores indicating higher resilience.

Depression was screened using the BDI-21. This is a self-report scale, consisting of 21 items assessing the presence and severity of depressive symptoms scoring on a 4-point Likert scale (from 0 to 3 ), over the last two weeks. According to the total score (0 to 63 ), the scores of 0-10 indicate normal results, and scores over 18 represent overt depression and the need for medical intervention (Mashhadi, Shakiba, \& Zakeri, 2013).

Emotion-related questions included 3 sections, including primary emotion, emotion regulation efforts, and emotion regulation strategies (English et al., 2017). The first section considered the primary negative emotions that participants were dominantly experienced facing with COVID-19-related news as an emotional stimulus. A list of negative emotions divided into High Arousal (HA) emotions (including anxiety and anger) and Low Arousal (LA) emotions (including homesickness, loneliness, sadness, fatigue, and disappointment) was provided and the participants were asked to select one of them (Hoyt, Craske, Mineka, \& Adam, 2015). They could choose "none" if they did not experience any of them. We also considered a section for participants to write about other emotions that were not provided in the list. The second section asked participants whether they often attempted to influence the negative emotions (selected in the previous section) and in the case of "Yes" response, they were guided to the third section. In the third section, the participants were provided with a list of strategies (including primary control, secondary control, disengagement, and involuntary engagement) that they often applied to control their emotions (Bettis et al., 2019). Primary control strategies included problemsolving ("I tried to find proper solutions to take care of myself and my family") and social sharing ("I tried to share my emotions and asking others for advice"), secondary control strategies included reappraisal ("I tried to think about a situation differently in order to change my mood") and distraction ("I shifted my attention away from what was making me emotional"), disengagement included suppression ("I tried not to reflect an emotion I felt inside"), and involuntary engagement included rumination ("I repeatedly thought about the COVID-19 issues and could not stop thinking for a moment") (English et al., 2017). The participants were also asked to rate the extent to which they regulated their primary emotions using each strategy on a 7-point Likert scale, rang- ing from "1 (not at all) to 7 (Always)". Scores of the strategies were calculated separately.

\subsection{Statistical analysis}

Mean and standard deviation were used to describe the basic characteristics of the participants in terms of sociodemographic, resilience, and depression scores, as well as emotion-related data. To compare emotion-related variables between the two groups (with high or low personal relevance), we used the Chi-squared test with $\mathrm{p}$ $<0.05$ considered statistically significant. We also used the point biserial correlation coefficient to explore the relationships between resilience scores and a dichotomized pattern of ER strategies (single or integrated approach). Statistical analyses were performed using SPSS 23.

\section{Results}

Of the 617 participants who completed the online survey, 104 respondents (16.8\%) were excluded because they obtained a score of over 18 on the BDI-21 scale. Overall, 443 participants were included in the analyses. Table 1 provides an overview of the descriptive characteristics of the participants.

The overview of the participants' responses to emotionrelated questions is provided in Table 2 . The primary negative emotions experienced facing COVID-19-related news were anxiety $(55.8 \%)$, homesickness $(8.4 \%)$, and sadness $(8.1 \%)$, respectively. Regardless of the type of primary emotions, the majority of participants (86.4\%) attempted to influence their negative emotions using ER strategies. Among 5.23 different ER strategies, problemsolving (mean score $=5.17$ ) and distraction (mean score $=$ 4.15), were the most frequently used strategies.

To explore the effect of personal relevance of the COVID-19-related news (as a family member of an infected or a deceased person or as a medical staff member engaged to fight against COVID-19), we compared the two groups of high personal relevance and low personal relevance regarding primary experienced emotions, as well as the frequently used ER strategies. It should be noted that both groups were homogenized in terms of mean age, gender, mean of the quarantine period, and the mean BDI-21 score $(p>0.05)$. We included the participants who attempted to influence their emotions $(n=386)$.

To reduce the number of variables, we used HA/LA classifications for primary experienced emotions, and concerning ER strategies, we identified the most frequent pattern for each participant in terms of single (only 
Table 1. Descriptive characteristics of the participants $(n=443)$

\begin{tabular}{|c|c|c|c|}
\hline & Variabels & & Mean \pm SD/ No. (\%) \\
\hline & Mean age & & $29.11(9.46)$ \\
\hline \multirow{2}{*}{ Gender } & & Female & $362(81.7)$ \\
\hline & & Male & $81(18.3)$ \\
\hline \multirow{4}{*}{ Educational level } & & High school & $27(6.1)$ \\
\hline & & Diploma & $71(16)$ \\
\hline & & Bachelor & $156(35.2)$ \\
\hline & & Master or high & $189(42.7)$ \\
\hline \multirow{6}{*}{ Number of family members } & & One & $18(4.1)$ \\
\hline & & Two & $100(22.2)$ \\
\hline & & Three & $117(26.4)$ \\
\hline & & Four & $167(37.7)$ \\
\hline & & Above four & $41(9.3)$ \\
\hline & & Mean of quarantine period (day) & $53.54(27.01)$ \\
\hline \multirow{4}{*}{ Personal relevance } & & High & $194(43.8)$ \\
\hline & & Low & $249(56.2)$ \\
\hline & & Mean BDI score & $7.96(4.67)$ \\
\hline & & Mean RS score & $56.77(7.03)$ \\
\hline
\end{tabular}

BDI: Beck Depression Inventory; RS: Resilience Scale

NEUR:SCIENCE

Table 2. Descriptive summary of the participants' responses to emotion-related questions $(n=443)$

\begin{tabular}{|c|c|c|}
\hline \multicolumn{2}{|c|}{ Variabels } & \multirow{2}{*}{$\frac{\text { No. }(\%) / \text { MeantSD }}{247(55.8)}$} \\
\hline & Anxiety & \\
\hline Primary HA emotions & & \\
\hline & Anger & $25(5.6)$ \\
\hline \multirow{7}{*}{ Primary LA emotions } & Homesickness & $37(8.4)$ \\
\hline & Loneliness & $1(0.2)$ \\
\hline & Sadness & $36(8.1)$ \\
\hline & & \\
\hline & Fatigue & $16(3.6)$ \\
\hline & Disappointment & $19(4.3)$ \\
\hline & None of the HA or LA emotions & $62(14)$ \\
\hline \multirow{2}{*}{ Attempt to influence primary emo } & Yes & $386(87.1)$ \\
\hline & No & $57(12.9)$ \\
\hline \multirow{3}{*}{ Primary control strategy } & Problem-solving & $5.23 \pm 2.28$ \\
\hline & & \\
\hline & Social sharing & $3.49 \pm 2.12$ \\
\hline \multirow{3}{*}{ Secondary control strategy } & Reappraisal & $4.00 \pm 2.26$ \\
\hline & & \\
\hline & Distraction & $4.15 \pm 2.53$ \\
\hline Disengagement & Suppression & $2.89 \pm 1.94$ \\
\hline Involuntary engagement & Rumination & $2.63 \pm 1.75$ \\
\hline
\end{tabular}

HA: High Arousal; LA: Low Arousal; ER: Emotion Regulation

NEUR:SCIENCE 
one strategy had the highest score) or integrated (more than one strategy had the highest scores) approach. We found that in the group with high personal relevance, HA and LA emotions were $61.9 \%$ and $25.7 \%$, respectively, whereas, in the group with low personal relevance, they obtained $60.6 \%$ and $25.4 \%$, respectively. A Chi-square test used to compare the primary emotions indicated no significant difference $\left(\mathrm{X}^{2}=0.006, \mathrm{p}=0.51\right)$. It means that both groups have experienced negative emotions equally independent of the personal relevance variable. Similarly, the patterns of frequently used ER strategies were identical between the two groups, as the primary control strategies (as a single approach) and the combination of primary and secondary control strategies were rated as the most frequently used strategies in the face of COVID-19-related news ( single approach $=35.8 \%$, integrated approach $=26.5 \%$ for the group with a high personal relevance; single $=34.1 \%$, integrated approach $=21.3 \%$ for the group with a low personal relevance). The Chi-square test indicated no significant difference between the groups $\left(\mathrm{X}^{2}=0.14, \mathrm{p}=0.39\right)$. Therefore, the pattern of frequently used ER strategies was similar between the groups independent of personal relevance. Moreover, we used the point biserial correlation to explore the relationship between resilience scores of the participants (who attempted to influence their emotions), and also divided ER strategies (single of integrated approach). The obtained significant positive correlation ( $r b p=0.15, p=0.01)$ indicated that those who used the integrated approach of ER strategies (including primary and secondary control) scored significantly higher on resilience scale (Mean=58.40, $\mathrm{SD}=6.21$ ) than those who used the single approach (primary control) $(\mathrm{Mean}=56.53, \mathrm{SD}=6.56)$.

\section{Discussion}

In the present study, we found that anxiety was the most common primary negative emotion that was experienced by individuals who regularly read the news on COVID-19 during the early stages after the first affected cases were reported in Iran (Abdi, 2020). Our results indicated that most of the individuals $(87.1 \%)$ participated in our survey, attempted to influence their emotions using ER strategies. According to the self-report data, problem-solving and distraction achieved the highest scores, whereas rumination and suppression had the lowest scores.

Moreover, to find the role of personal relevance, we divided participants into two groups in terms of high and low personal relevance based on the participant's direct involvement with issues related to COVID-19. Interestingly, both groups showed the same pattern in experiencing negative emotions, as well as using ER strategies. In both groups, HA emotions, including anxiety and anger were more common than LA emotions, such as homesick- ness, loneliness, sadness, fatigue, and disappointment. This finding indicates that regardless of being affected or not affected by COVID-19 news, news media may elicit similar negative emotions that can be perceived as personally relevant by both groups. Also, previous studies have indicated that being exposed to emotional programs provided by media may result in intense emotional responses (Oh, Lee, \& Han, 2020) probably reflecting as anxiety, particularly while facing uncertain existential threats (Lazarus et al., 1991). In terms of the frequent pattern of applied ER strategies, both groups used primary control strategies (including problem-solving and social sharing) and the combination of primary and secondary control strategies. Therefore, regardless of personal relevance, the negative emotions elicited by the news on COVID-19 were controlled similarly.

Although measuring the effectiveness of these applied ER strategies in controlling the negative emotions was not considered in this study, we found that individuals who used the integrated approach (including primary and secondary control strategies), obtained a significantly higher score from the resilience scale, indicating that resilient individuals who are able to adapt well in the face of adversity, trauma, tragedy, threats or stressful condition, use multiple ER strategies (Southwick et al., 2016). Using an integrative approach to regulate emotions supports this fact about resilient people who are characterized by their abilities to respond flexibly against changing emotional context, as we have experienced while reading the news during the COVID-19 crisis.

This study had several limitations. First, the used samples included volunteer adults and they are not representative of the general population. Second, we did not assess anxiety to screen participants, because, in many studies, selfassessment tools used to identify anxiety disorders often show less favorable psychometric characteristics (Rose \& Devine., 2014). Finally, we relied on participants' reports regarding their emotions and emotion regulation strategies; therefore, our findings may be affected by the personal recall. Future studies may benefit from using more precise measures.

Overall, the findings from this study indicated that emotional reactions to the events, such as the COVID-19 crisis, maybe experienced similarly ssssssssby different groups of people in society regardless of personal relevance. Future studies may benefit from immediate and long-term monitoring of an individual's experiences in the face of media offering emotional content, particularly the negative media content. 


\section{Ethical Considerations}

\section{Compliance with ethical guidelines}

All ethical principles were considered in this article.

Funding

This study received no external funding resources.

\section{Authors' contributions}

Hamidein designed the survey and collected data: Zahra Hamidein; wrote the paper and analyzed data: Tara Rezapour, edited the paper: Javad Hatami.

\section{Conflict of interest}

The authors declared no conflict of interest.

\section{References}

Abdi, M. (2020). Coronavirus disease 2019 (COVID-19) outbreak in Iran: Actions and problems. Infection Control and Hospital Epidemiology, 41(6), 754-5. [DOI:10.1017/ice.2020.86] [PMID] [PMCID]

Basch, C. H., Hillyer, G. C., Meleo-Erwin, Z., Mohlma, J., Cosgrove, A., \& Quinones, N. (2020). News coverage of the COVID-19 pandemic: Missed opportunities to promote health sustaining behaviors. Infection, Disease $\mathcal{E}$ Health. [Published Online] [DOI:10.1016/j.idh.2020.05.001] [PMID] [PMCID]

Bayer, M., Ruthmann, K., \& Schacht, A. (2017). The impact of personal relevance on emotion processing: Evidence from event-related potentials and pupillary responses. Social Cognitive and Affective Neuroscience, 12(9), 1470-9. [DOI:10.1093/ scan/nsx075] [PMID] [PMCID]

Bernstein, K. T., Ahern, J., Tracy, M., Boscarino, J. A., Vlahov, D., \& Galea, S. (2007). Television watching and the risk of incident probable posttraumatic stress disorder: A prospective evaluation. The Journal of Nervous and Mental Disease, 195(1), 41-7. [DOI:10.1097/01.nmd.0000244784.36745.a5] [PMID]

Bettis, A. H., Henry, L., Prussien, K. V., Vreeland, A., Smith, M., \& Adery, L. H., et al. (2019). Laboratory and self-report methods to assess reappraisal and distraction in youth. Journal of Clinical Child \& Adolescent Psychology, 48(6), 855-65. [DOI:10.1 080/15374416.2018.1466306] [PMID] [PMCID]

Block, J., \& Kremen, A. M. (1996). IQ and ego-resiliency: Conceptual and empirical connections and separateness. Journal of Personality and Social Psychology, 70(2), 349-61. [DOI:10.1037/0022-3514.70.2.349] [PMID]

Bodas, M., Siman-Tov, M., Peleg, K., \& Solomon, Z. (2015). Anxiety-inducing media: The effect of constant news broadcasting on the well-being of Israeli television viewers. Psychiatry, 78(3), 265-76. [DOI:10.1080/00332747.2015.1069658] [PMID]
Boehme, S., Biehl, S. C., \& Mühlberger, A. (2019). Effects of differential strategies of emotion regulation. Brain Sciences, 9(9), 225. [DOI:10.3390/brainsci9090225] [PMID] [PMCID]

Brans, K., Koval, P., Verduyn, P., Lim, Y. L., \& Kuppens, P. (2013). The regulation of negative and positive affect in daily life. Emotion, 13(5), 926-39. [DOI:10.1037/a0032400] [PMID]

Butler, E. A., Egloff, B., Wlhelm, F. H., Smith, N. C., Erickson, E. A., \& Gross, J. J. (2003). The social consequences of expressive suppression. Emotion, 3(1), 48-67. [DOI:10.1037/15283542.3.1.48] [PMID]

Cooper, C. P., \& Roter, D. L. (2000). "If it bleeds it leads"? Attributes of TV health news stories that drive viewer at tention. Public Health Reports, 115(4), 331-8. [DOI:10.1093/ $\mathrm{phr} / 115.4 .331]$ [PMID] [PMCID]

de Hoog, N., \& Verboon, P. (2020). Is the news making us unhappy? The influence of daily news exposure on emotional states. British Journal of Psychology, 111(2), 157-73. [DOI:10.1111/ bjop.12389] [PMID] [PMCID]

Doré, B. P., Silvers, J. A., \& Ochsner, K. N. (2016). Toward a personalized science of emotion regulation. Social and Personality Psychology Compass, 10(4), 171-87. [DOI:10.1111/spc3.12240] [PMID] [PMCID]

English, T., Lee, I. A., John, O. P., \& Gross, J. J. (2017). Emotion regulation strategy selection in daily life: The role of social context and goals. Motivation and Emotion, 41(2), 230-42. [DOI:10.1007/s11031-016-9597-z] [PMID] [PMCID]

Gao, J., Zheng, P., Jia, Y., Chen, H., Mao, Y., \& Chen, S., et al. Mental health problems and social media exposure during COVID-19 outbreak. The Lancet. [Published Online] [DOI:10.2139/ssrn.3541120]

Gross, J. J. (1998). Antecedent- and response-focused emotion regulation: Divergent consequences for experience, expression, and physiology. Journal of Personality and Social Psychology, 74(1), 224-37. [DOI:10.1037/0022-3514.74.1.224] [PMID]

Gutentag, T., Halperin, E., Porat, R., Bigman, Y. E., \& Tamir, M. (2017). Successful emotion regulation requires both conviction and skill: Beliefs about the controllability of emotions, reappraisal, and regulation success. Cognition and Emotion 31(6), 1225-33. [DOI:10.1080/02699931.2016.1213704] [PMID]

Haroon, O., \& Rizvi, S. A. R. (2020). COVID-19: Media coverage and financial markets behavior- a sectoral inquiry. Journal of Behavioral and Experimental Finance, 27, 100343. [DOI:10.1016/j. jbef.2020.100343] [PMID] [PMCID]

Hoyt, L. T., Craske, M. G., Mineka, S., \& Adam, E. K. (2015) Positive and negative affect and arousal: Cross-sectional and longitudinal associations with adolescent cortisol diurnal rhythms. Psychosomatic Medicine, 77(4), 392-401. [DOI:10.1097/ PSY.0000000000000178] [PMID] [PMCID]

Kashdan, T. B., Goodman, F. R., Mallard, T. T., \& DeWall, C. N. (2016). What triggers anger in everyday life? Links to the intensity, control, and regulation of these emotions, and personality traits. Journal of Personality, 84(6), 737-49. [DOI:10.1111/ jopy.12214] [PMID]

Kay, S. A. (2016). Emotion regulation and resilience: Overlooked connections. Industrial and Organizational Psychology, 9(2), 4115. [DOI:10.1017/iop.2016.31] 
Klohnen, E. C. (1996). Conceptual analysis and measurement of the construct of ego-resiliency. Journal of Personality and Social Psychology, 70(5), 1067-79. [DOI:10.1037/0022-3514.70.5.1067] [PMID]

Kobylińska, D., \& Kusev, P. (2019). Flexible emotion regulation: How situational demands and individual differences influence the effectiveness of regulatory strategies. Frontiers in Psychology, 10, 72. [DOI:10.3389/fpsyg.2019.00072] [PMID] [PMCID]

Lazarus, R. S. (1991). Progress on a cognitive-motivational-relational theory of emotion. American Psychologist, 46(8), 819-34. [DOI:10.1037/0003-066X.46.8.819] [PMID]

Lennarz, H. K., Hollenstein, T., Lichtwarck-Aschoff, A., Kuntsche, E., \& Granic, I. (2019). Emotion regulation in action: Use, selection, and success of emotion regulation in adolescents' daily lives. International Journal of Behavioral Development, 43(1), 1-11. [DOI:10.1177/0165025418755540] [PMID] [PMCID]

Li, S., Wang, Y., Xue, J., Zhao, N., \& Zhu, T. (2020). The impact of COVID-19 epidemic declaration on psychological consequences: A study on active Weibo users. International Journal of Environmental Research and Public Health, 17(6), 2032. [DOI:10.3390/ijerph17062032] [PMID] [PMCID]

Mashhadi, M. A., Shakiba, M., \& Zakeri, Z. (2013). Evaluation of depression in patients with cancer in South of Iran (Zahedan). Iranian Journal of Cancer Prevention, 6(1), 12-6. [PMID] [PMCID]

Oh, S. H., Lee, S. Y., \& Han, C. (2020). The effects of social media use on preventive behaviors during infectious disease outbreaks: The mediating role of self-relevant emotions and public risk perception. Health Communication. [Published Online] [DOI:10.1080/10410236.2020.1724639] [PMID]

Restubog, S. L. D., Ocampo, A. C. G., \& Wang, L. (2020). Taking control amidst the chaos: Emotion regulation during the COVID-19 pandemic. Journal of Vocational Behavior, 119, 103440. [DOI:10.1016/j.jvb.2020.103440] [PMID] [PMCID]

Richards, J. M., \& Gross, J. J. (2000). Emotion regulation and memory: The cognitive costs of keeping one's cool Journal of Personality and Social Psychology, 79(3), 410-24. [DOI:10.1037/0022-3514.79.3.410] [PMID]

Rose, M., \& Devine, J. (2014). Assessment of patient-reported symptoms of anxiety. Dialogues in Clinical Neuroscience, 16(2), 197-211. [PMID] [PMCID]

Smith, C. A., \& Kirby, L. D. (2000). Consequences require antecedents: Toward a process model of emotion elicitation. In J. P. Forgas (Ed.), Feeling and thinking: The role of affect in social cognition (pp. 83-106). New York: Cambridge University Press. https://www.researchgate.net/publication/229059959

Southwick, S. M., Bonanno, G. A., Masten, A. S., Panter-Brick, C., \& Yehuda, R. (2014). Resilience definitions, theory, and challenges: Interdisciplinary perspectives. European Journal of Psychotraumatology, 5. [DOI:10.3402/ejpt.v5.25338] [PMID] [PMCID]

Southwick, S. M., Sippel, L., Krystal, J., Charney, D., Mayes, L., \& Pietrzak, R. (2016). Why are some individuals more resilient than others: The role of social support. World Psychiatry, 15(1), 77-9. [DOI:10.1002/wps.20282] [PMID] [PMCID]
Szabo, A., \& Hopkinson, K. L. (2007). Negative psychological effects of watching the news in the television: Relaxation or another intervention may be needed to buffer them! International Journal of Behavioral Medicine, 14(2), 57-62. [DOI:10.1007/ BF03004169] [PMID]

Thompson, R. A. (1994). Emotion regulation: A theme in search of definition. Monographs of the Society for Research in Child Development, 59(2-3), 25-52. [DOI:10.1111/j.1540-5834.1994. tb01276.x]

Wagnild, G. M. (2009). The Resilience Scale user's guide: For the U.S. English version of the Resilience Scale and the 14-item Resilience Scale (RS-14). Montana: Resilience Center. [Published Online] https://books.google.com/ books?id=A_7VSAAACAAJ\&dq

Wagnild, G. M., \& Young, H. M. (1993). Development and psychometric evaluation of the resilience scale. Journal of Nursing Measurement, 1(2), 165-78. [PMID]

Webb, T. L., Miles, E., \& Sheeran, P. (2012). Dealing with feeling: A meta-analysis of the effectiveness of strategies derived from the process model of emotion regulation. Psychological Bulletin, 138(4), 775-808. [DOI:10.1037/a0027600] [PMID]

Wolin, S. J., \& Wolin, S. (1993). Bound and determined: Growing up resilient in a troubled family. New York: Villard Press. 